ISBN 82-553-0606-4

Applied Mathematics
No 2

30 April 1986

\title{
ON RADIATED AND SCATTERED WAVES \\ FROM A SUBMERGED ELLIPTIC CYLINDER \\ IN A UNIFORM CURRENT
}

by

A. Mo and E. Palm

PREPRINT SERIES - Matematisk institutt, Universitetet i Oslo 


\begin{abstract}
The two-dimensional radiation problem and diffraction problem are discussed for submerged elliptic cylinders when a current is present. It is shown that the impact of the current on the wave amplitudes and wave forces are large. The singularity in the problem, corresponding to a wave travelling upstream with a group velocity equal to the speed of the current, is examined. As expected, this singularity influences the motion strongly. We find, however, that the amplitudes and forces remain finite.
\end{abstract}


dingly, when one harmonic wave is diffracted by a submerged body, normally three new waves are generated when $\tau<1 / 4$ and one new wave when $\tau>1 / 4$.

The actual wave problem has been discussed in a recent paper by Grue and Palm (1985) (hereafter called I) for a submerged body of form as a circular cylinder. It turns out, however, that the circular cylinder is no typical representative for a submerged body of arbitrary two-dimensional form. For example, in the diffraction problem it is found that a circular cylinder, in contrast to other bodies, generates only one new wave also for $\tau<1 / 4$. In this paper we shall consider a submerged elliptical cylinder. The obvious advantage of this contour is that by changing the eccentricity we obtain bodies varying from a circle to a flat plate.

It will be clear that the case $\tau=1 / 4$ is of special interest in this problem. Physically it corresponds to that $\sigma$ is tuned so that the wave travelling upstream has group velocity equal to the speed of the current. It seems to be generally accepted in the litterature that this case will lead to infinite wave amplitudes and hence infinite wave forces. We obtain, however, that the wave amplitudes and wave forces are finite for all values of $\tau$. It is shown, however, that the motion has certain pecularities near $\tau=$ $1 / 4$. Thus in the diffraction problem for an incoming $k_{2}$ wave (defined as a wave,travelling upstream) the transmitted wave tends towards zero as $\tau \rightarrow 1 / 4$ whereas a reflected $k_{1}$ wave (defined in section 2) is generated, travelling downstream with an amplitude equal to the incoming wave. The corresponding result is obtained for an incoming $k_{1}$ wave with the $k_{1}$ wave and the $k_{2}$ wave changing roles. Due to this fact we may close to $\tau=1 / 4$ obtain practically speaking, total reflection. We find also that the 
where $j$ is the imaginary unit, and $R_{j} j$ denotes the real part (with respect to $j$ ). Both $\chi$ and $\phi$ satisfy the Laplacian equation

$$
\nabla^{2} \chi=0 ; \quad \nabla^{2} \phi=0
$$

Assume that the cylinder is oscillating with its centre at

$$
x=\operatorname{Re}_{j} \xi_{x} \exp (j \sigma t), \quad y+d=\operatorname{Re}_{j} \xi_{y} \exp (j \sigma t)
$$

and rotating with an angular displacement $\vec{~}$. The body boundary conditions applied at the mean position of the body surface $S$ is

$$
\frac{\partial x}{\partial n}=-n x
$$

where $\frac{\partial}{\partial n}$ denotes the normal derivative, and

$$
\frac{\partial \phi}{\partial n}=\vec{n} \cdot[j \sigma \vec{\alpha}+U(\vec{\alpha} \cdot \nabla) \nabla \chi]+U(\vec{\Omega} \times \vec{n}) \cdot \nabla(\chi+x)
$$

(see Newman 1978, eq. 3.28). Here

$$
\vec{\alpha}=\vec{\xi}+\vec{a} \times \vec{r}, \quad \vec{\xi}=\left(\xi_{x}, \xi_{y}\right)
$$

$\vec{n}$ is the normal vector of the body, and $\vec{r}$ is the vector from the centre of the ellipse to a point on the surface (see fig.1).

The linearized boundary condition at $y=0$ is obtained by combining the dynamic and kinematic boundary conditions which gives

$$
\begin{aligned}
& U^{2} \frac{\partial^{2} \chi}{\partial x^{2}}+g \frac{\partial \chi}{\partial x}=0 \\
& \left(j \sigma-U \frac{\partial}{\partial x}\right)^{2} \phi+g \frac{\partial \phi}{\partial y}=0
\end{aligned}
$$

To solve (2.2) with the proper boundary conditions we shall transform the mathematical problem to an integral equation. This may be achieved by expressing $\phi$ (and $\chi$ ) as a source distribution over the boundary of the submerged body. For a detailed derivation of the integral equation, we refer to $I$, and here only give the necessary 
and a bar denotes complex conjugate. With

$$
\nu=\frac{\sigma^{2}}{g}, \tau=\frac{U \sigma}{g}
$$

the four wave numbers are defined by

$$
k_{1,2}=\frac{\nu}{2 \tau^{2}}(1-2 \tau \pm \sqrt{1-4 \tau}), k_{3,4}=\frac{\nu}{2 \tau^{2}}(1+2 \tau \pm \sqrt{1+4 \tau})
$$

For $\tau>1 / 4, k_{1}$ and $k_{2}$ become complex in $i$.

Before proceeding further, let us discuss shortly the farfield motion due to the concentrated source. It is seen from $(2.12)-(2.16)$ that for $\tau>1 / 4$ the solution consists of four waves, viz one wave with wave number $k_{2}$ at $x=\infty$ and three waves with wave numbers $k_{1}, k_{3}, k_{4}$ at $x=-\infty$. For $\tau>1 / 4$ the solution consists of no waves at $x=\infty$ and two waves with wave numbers $k_{3}, k_{4}$ at $x=-\infty$.

The various wave numbers are found as solutions of i) $\sigma=\mathrm{Uk} \pm(\mathrm{gk})^{\frac{1}{2}}\left(\mathrm{k}=\mathrm{k}_{4}, \mathrm{k}_{3}\right)$ and $\left.\mathrm{ii}\right) \quad \sigma=(\mathrm{gk})^{\frac{1}{2}}-\mathrm{Uk} \quad\left(\mathrm{k}=\mathrm{k},, \mathrm{k}_{2}\right)$ where $\sigma$ is positive and known. The four solutions are indicated in figure 2. It is seen from the figure that, in order to get four waves, $\sigma$ must be less than a certain maximal value, i.e. $\tau<1 / 4$. Furthermore, in the relative frame of reference, both the $k_{1}$ wave and the $\mathrm{k}_{2}$ wave have positive phase velocities which are larger than $U$. The $k_{1}$ wave has, however, a group velocity less than $U$, and is therefore located downstream. The $k_{2}$ wave has a group velocity larger than $\mathrm{U}$, and is located upstream. The $\mathrm{k}_{3}$ wave has positive phase velocity smaller than $\mathrm{U}$, and the $\mathrm{k}_{4}$ wave has negative phase velocity. These two waves are therefore located downstream. 
Equation (2.17) is a (non-singular) Fredholm equation of second kind. The equation is solved by using a collocation method with cubic splines. A very good convergence is generally obtained by using 20-35 collocation points equally spaced. The more slender the body is, the more points are needed. The accuracy is about $1 \%$ or better in all results presented in this paper.

We shall be especially interested in the far-field. By contour integration we obtain from (2.11), applying (2.12)-(2.14), that

$$
\begin{gathered}
\lim _{x \rightarrow \infty} f_{1}(z)=A_{2} \exp \left(-i k_{2} z\right) \\
\lim _{x \rightarrow-\infty} f_{1}(z)=A_{1} \exp \left(-i k_{1} z\right)+A_{3} \exp \left(-i k_{3} z\right)-A_{4} \exp \left(-i k_{4} z\right)
\end{gathered}
$$

where

$$
\begin{aligned}
& A_{1,2}=i(1-i j) \frac{1}{\sqrt{1-4 \tau}} \int_{S} \gamma(s) \exp \left(i k_{1,2} \zeta(s)\right) d s \\
& A_{3,4}=i(1+i j) \frac{1}{\sqrt{1+4 \tau}} \int_{S} \gamma(s) \exp \left(i k_{3,4} \zeta(s)\right) d s
\end{aligned}
$$

For an elliptic contour it is appropriate to write the equation for the ellipse on parameter form as

$$
\zeta(\theta)=R \cos (\theta)+i b \operatorname{in}(\theta)-i(d+b)
$$

and using $\theta$ as variable instead of $s$. (For definitions of $R, b$, d and $\theta$, see fig. 1.)

3. THE OSCILLATING ELLIPTIC CYLINDER

Let us consider an elliptic cylinder oscillating in sway, heave and roll. The right hand side of the integral equation (2.17) is now given by $(2.19)$ and $(2.5)$ with $f_{0}$ chosen as zero. Thus $h\left(s^{\prime}\right)$ is given by 
and $U=0$. As expected, the maximum relative amplitude is obtained in heave and for the smallest value of $b / R, i . e \cdot b / R=0.05$. For later reference we note that the maximum amplitude in heave for $b / R=0.3$ occurs for $\nu R \approx 1.2$.

To examine the effect of increasing the depth of the cylinder, $d / R$ is in fig. 4 chosen as 2 (the only case where $d / R$ is not one). It is noticed that the maximum amplitude now is about $40 \%$ of the maximum amplitude for $\mathrm{d} / \mathrm{R}=1$.

In figs $5 a, 5 b, 5 c$ are displayed the relative amplitudes for sway, heave and roll, respectively for the Froude number $\mathrm{Fr}=\mathrm{U} /(\mathrm{gR})^{\frac{1}{2}}$ equal to 0.2. The corresponding curves for $\mathrm{Fr}=0.4$ are shown in figs $6 a, 6 b, 6 c$. We notice that for $\operatorname{Fr}=0.2$ the $k_{3}$ wave has a vanishing amplitude. This is also true for the $k_{1}$ wave except very close to $\tau=1 / 4$ (corresponding to $\nu R=1.5625$ ). Hence, practically speaking, for $\nu \mathrm{R}$ less than 1.5625 , the motion for $\mathrm{Fr}=0,2$ consists of one wave at $\mathrm{x}=\infty$ (the $\mathrm{k}_{2}$ wave) and one wave at $\mathrm{x}=-\infty$ (the $\mathrm{k}_{4}$ wave). For larger values of $v R$ only the $\mathrm{k}_{4}$ wave occurs.

For $\mathrm{Fr}=0.4$ three waves occur when $\tau<1 / 4$, viz the $\mathrm{k}$, wave, the $\mathrm{k}_{2}$ wave and the $\mathrm{k}_{4}$ wave. For $\mathrm{Fr}=0.7$ and $\mathrm{Fr}=1.0$ we find that all four waves have appriciable amplitudes. To get an idea of which of the waves that are important for a given Froude number, and for which values of $v R$ these waves obtain their maximum amplitudes, we examine first the corresponding values for $\mathrm{Fr}=0$. As an example, let us consider the heave motion for Fr $=0.2$ (fig. $5 \mathrm{~b}$ ). We have noticed above that for $\operatorname{Fr}=0$ the maximum amplitude for heave occurs when $v R=1.2$ (fig. $3 b$ ). This corresponds to a far-field motion with $k R=1.2$ where $k$ is the wave number. It seems reasonable to expect that also for $\operatorname{Fr} \neq 0$, 


$$
\begin{aligned}
& \delta \gamma\left(s^{\prime}\right)+ \\
& \frac{1}{\pi} \int_{s} \gamma(s) \operatorname{Im}_{i}\left\{\operatorname { e x p } ( i \beta ( s ^ { \prime } ) ) ( i + j ) \left[k_{1} \int_{\infty}^{\zeta\left(s^{\prime}\right)} \frac{\exp \left(-i k_{1}\left(\zeta\left(s^{\prime}\right)-u\right)\right) d u}{u-\overline{\zeta(s)}}\right.\right. \\
& \left.\left.-k_{2} \int_{-\infty}^{\zeta\left(s^{\prime}\right)} \frac{\exp \left(-i k_{2}\left(\zeta\left(s^{\prime}\right)-u\right)\right) d u}{u-\overline{\zeta(s)}}\right]\right\} \\
& =0(\delta)
\end{aligned}
$$

To the same order of accuracy we may set $k_{1}=k_{2}=k_{\text {, say. The }}$ inner integral is then evaluated by contour integration. Thereby

$$
\begin{aligned}
& \delta \gamma\left(s^{\prime}\right)- \\
& \begin{aligned}
2 \operatorname{kIm}_{i}\left\{( i + j ) \operatorname { e x p } \left(i \beta\left(s^{\prime}\right)-\right.\right. & \left.i k \zeta\left(s^{\prime}\right)\right) \int_{S} \gamma(s) \exp (i k \overline{\zeta(s)} d s\} \\
& =0(\delta)
\end{aligned}
\end{aligned}
$$

We notice that the integral in (2.23), with $k_{1}, k_{2}=k_{\text {, is exactly }}$ the same as the integral in (3.5). Hence, if $\gamma$ remains finite, we deduce that $A_{1,2}$ is finite in the limit, in spite of the factor $\delta^{-1}$. This result is valid for an arbitrary smooth body.

\subsection{The forces}

In many practical problems where a body is oscillating, for example due to incoming waves, it is important to know the magnitude of the damping force. Usually this force is mainly due to viscous effects. However, if the body is located near the free surface, the damping due to the radiated waves, may be of practical importance. To examine the magnitude of this force, we first note that it can be written as

$$
F=D \sin \sigma \tau
$$


fig. 7 that it is important to take into account the effect of the current.

It may be of interest to compare the wave damping with the viscous drag for the restrained body. The latter may for an elliptic cylinder be written

$$
F=\frac{1}{2} C_{D} \rho U^{2} 2 b
$$

where $C_{D}$ is the drag coefficient. The damping force may according to fig. 7 be written

$$
D=\lambda g \rho R \varepsilon
$$

where $\lambda$ is of order unity or smaller. Hence

$$
\frac{\mathrm{D}}{\mathrm{F}}=\frac{\lambda}{\mathrm{C}_{\mathrm{D}} \mathrm{Fr} \mathrm{r}^{2}} \frac{\varepsilon}{\mathrm{b}}
$$

$C_{D}$ for various elliptic contours is discussed in Modern Developments In Fluid Dynamics (1938, p.415). A reasonable characteristic value for $b / R=0.3$ is $c_{D}=0.15$. Let us choose $\operatorname{Fr}=0.4$. The maximum value of $\lambda$ is then 0.9 , and the ratio between the maximum drag force and the viscous drag is

$$
\frac{D}{F} \approx 40 \frac{\varepsilon}{b}
$$

We see that even for very small $\varepsilon, D / F$ may be larger than unity. Obviously, when the depth $d$ of the cylinder is increased, the damping force decreases. We have examined the magnitude of this effect for the case $U=0$ by also computing the forces when $\mathrm{d} / \mathrm{R}=2.0$. The damping force for heave in this case is displayed in fig. 4. Comparing this with the result for the damping force for Fr $=0$ in fig. 7, we note that the maximum value in the deeper case is about $30 \%$ of the maximum value for $d / R=1.0$. 
$(2.24) \cdot \gamma$ is obtained by solving (2.17). The right hand side of this equation is now (2.19) with $\partial \phi / \partial n=0$ and $f_{0}(z)$ given by (4.3). In all examples below the distance between the mean free surface and the uppermost point of the ellipse is equal to $R$, as in almost all examples in section 3 . Furthermore, b/R is 0.3 , except in fig. 9 .

In the relative frame of reference, the incoming wave will be a wave travelling in either the same or in the opposite direction as the body. In the latter case the wave is what we have termed a $\mathrm{k}_{4}$ wave. In the first case the wave will be either a $k_{2}$ wave, $k_{1}$ wave or $k_{3}$ wave, depending on the magnitude of the wave number. From the discussion in section 2 it follows that a $k_{2}$ wave has a large wave length such that both the group velocity and phase velocity are larger than $U, a k_{1}$ wave has phase velocity larger and group velocity smaller than $U$ and $a k_{3}$ wave has both group velocity and phase velocity smaller than $U$.

\subsection{The far-field motion}

Let us first consider the case $U=0$. The amplitudes of the reflected waves are displayed in fig. 9 for various values of $b / R$. Since the amplitude of the reflected wave is zero for $b / R=1$ (Dean 1948), we expect this amplitude to increase for decreasing values of $b / R$. This is seen to be true, and for $b / R=0.05$ we find from the figure that the maximum reflected amplitude is about 0.3 times the incomig amplitude. This value may be compared with the result for the flat plate, resently discussed by Bjordal (1985). He obtains for $d / R=1$ that the maximum reflected amplitude is 0.294 times the incoming amplitude. 
Froude numbers a $k_{3}$ wave will be generated by an incoming $k_{4}$ wave. In this case we have no reflected wave, but the transmitted wave is splitted in two waves, viz a $k_{3}$ wave and a $k_{4}$ wave. In $\mathrm{fig} .11$ are shown the wave amplitudes for $\mathrm{Fr}=0.4$. In fig. 11 a the incoming wave is $a k_{1}$ wave and the generated waves is $a k_{2}$ wave and $a k_{4}$ wave. In $f i g .11 b$ the incoming wave is a $\mathrm{k}_{2}$ wave, and $\mathrm{a} \mathrm{k}_{4}$ wave and $\mathrm{a} \mathrm{k}_{1}$ wave are generated. In both figs $11 \mathrm{a}$ and $11 \mathrm{~b}$ the $\mathrm{k}_{4}$ wave are, however, almost negligible. The most characteristic feature of these two figures is that for an incoming $\mathrm{k}_{1}$ wave $\left(\mathrm{k}_{2}\right.$ wave $)$ the amplitude tends towards zero approaching $\tau=1 / 4$, whereas the generated $\mathrm{k}_{2}$ wave ( $\mathrm{k}_{1}$ wave) obtaines an amplitude for $\tau \rightarrow 1 / 4^{-}$equal to the amplitude of the incoming wave. This is always found to be true. An interpretation of this result is that $a k_{1}$ wave or $k_{2}$ wave will be strongly reflected near $\tau=1 / 4$ and as $\tau=1 / 4$ is approached, we obtain with a very good approximation total reflection. Another remarkable feature in $f i g .11 b$ is that $a_{1} / a_{0}$ may be larger than one, i.e. we have overreflection. The overreflection is even stronger in fig. 12, where the diffraction properties for $\operatorname{Fr}=0.7$ for an incoming $\mathrm{k}_{2}$ wave are displayed.

In fig. $11 \mathrm{c}$ the incoming wave is a $\mathrm{k}_{4}$ wave. For $\tau<1 / 4$ a $k_{1}$ wave and $a k_{2}$ wave are set up. Hence in this case the motion consists of an incoming wave travelling downstream (the $\mathrm{k}_{4}$ wave), a transmitted wave (the $\mathrm{k}_{4}$ wave), a reflected wave (the $\mathrm{k}_{2}$ wave) and a generated $k_{1}$ wave travelling downstream. For $\tau>1 / 4$ the $\mathrm{k}_{4}$ wave passes by the submerged body without noticing it. If the submerged elliptic cylinder is replaced by a circular cylinder, the wave motion for $\tau<1 / 4$ is very different. In this case the $\mathrm{k}_{1}$ wave and the $\mathrm{k}_{2}$ wave are exactly zero, as shown in $\mathrm{I}$. 
creasing Froude numbers for incoming waves travelling downstream and decreases for incoming waves travelling upstream.

For $\operatorname{Fr}=0.2$ and $\operatorname{Fr}=0.4$ we see that the maximum value of the first order horisontal force is about $0.4 \rho g R a_{0}$. The ratio between this force and the viscous drag (3.11) is

$$
\frac{\mathrm{X}_{1}}{\mathrm{~F}}=\frac{0.4}{\mathrm{C}_{\mathrm{D}} \mathrm{Fr}^{2}} \frac{\mathrm{a}_{0}}{\mathrm{~b}}
$$

Introducing $C_{D}=0.15$ and $F r=0.2$ we obtain that $x_{1} / F \approx 67 a_{0} / b$. The maximum value of the first order vertical force is $1.2 \mathrm{pgRa}_{0}$, obtained for $\mathrm{Fr}=0.4$ and incoming wave travelling downstream. The ratio between this force and the viscous drag is then $\mathrm{x}_{2} / \mathrm{F} \approx 200 \mathrm{a}_{0} / \mathrm{b}$.

\section{THE LEE-WAVE PROBLEM}

To solve the radiation problem, it is necessary to find the lee-wave potential $\chi$. The knowledge of the lee-wave solution is also important to examine the validity of our solution. A necessary condition for the linearized solution to be a good approximation, is that $\partial \chi / \partial x<1$. In fig. 14 is displayed $\max \left|\frac{\partial \chi}{\partial x}\right|$ at $x=-\infty$ when $b / R=0.3$. It is seen that $\max \left|\frac{\partial \chi}{\partial x}\right|$ is less than 0.1 for Froude numbers less than 0.6. For the sake of completeness we have also shown the amplitudes of the lee-waves for various parameters.

\section{SUMMARY AND CONCLUSION}

The two-dimensional radiation problem and diffraction problem are discussed for submerged elliptic cylinders. when a current is present. The amplitudes of the generated waves, the damping force, the first order horizontal force and the mean second order horizontal force are computed. It is found that the singularity at $\tau=1 / 4$ has a great influence on the magnitude of amplitudes and forces, but both the wave amplitudes and forces remain finite when 


\section{REFERENCES}

Bjordal, B. 1985 Wave reflection from a submerged flat plate. Master thesis, Institute of Mathematics, University of Oslo (in Norwegian).

Dagan, G. \& Miloh, T. 1982 Free surface flow past oscillating singularities. J. Fluid Mech. $120,139$.

Dean, W.R. 1948 On the reflection of surface waves by a circular cylinder. Proc. Camb. Phil. Soc. $44,483$.

Goldstein, S. (ed.) 1938 Modern developments in fluid dynamics, vol. 2, Oxford.

Grue. J. \& Palm, E. 1985 Wave radiation and wave diffraction from a submerged body in a uniform current. J. Fluid Mech. 151, 257 .

Haskind, M.D. 1954 On wave motion of a heavy fluid, Prikl. Mat. Mekh. 18, 15 .

Newman, J.N. 1961 The Damping of an oscillating Ellipsoid Near a Free Surface. J. Ship Research, $\underline{5}$, no. 3 .

Newman, J.N. 1978 The theory of ship motions. Adv.Appl.Math. 18, 221 .

Newman, J.N. 1980 Marine Hydrodynamics. The MIT press. 




Fig. 1 Geometry of the problem.

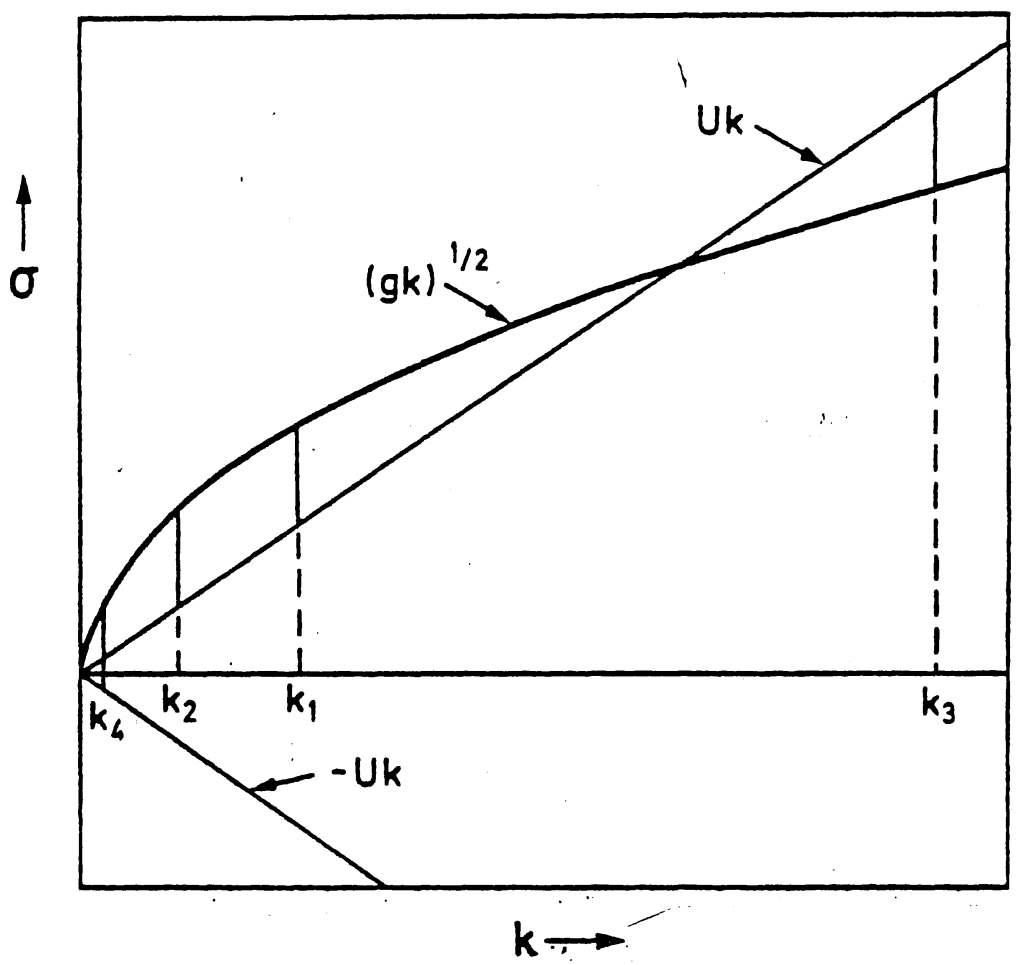

Fig. 2 The four wave numbers for given $\sigma$ and $U$. 


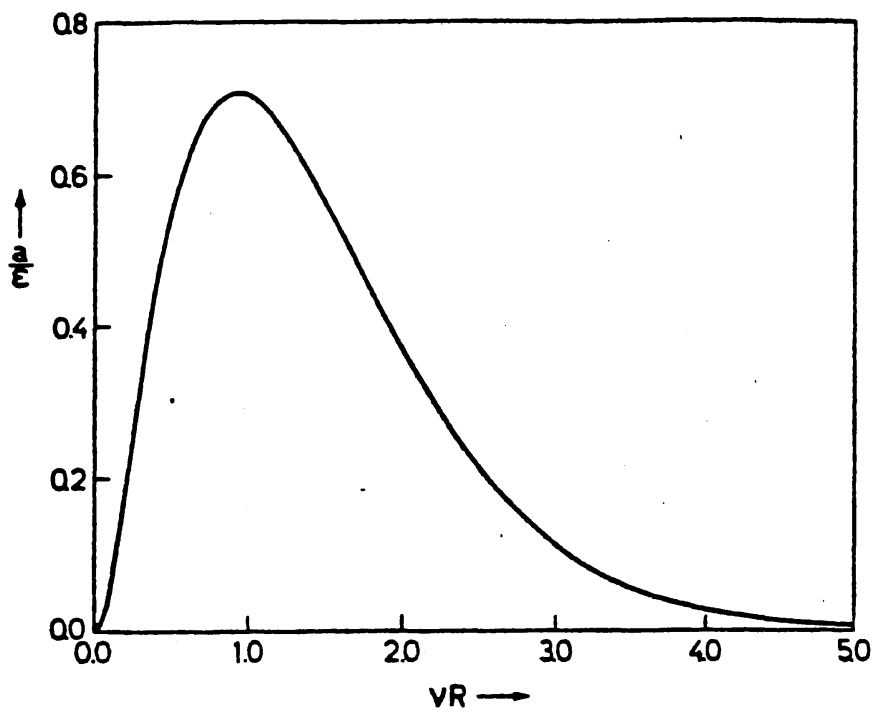

Fig. 3a. Amplitudes for radiated waves in sway or heave for a circle (b/F 1.0, $d / r=1.0), F r=0$.



Fig. 3c. Anplitudes for radiated waves in heave, sway and roll ( $b / R=0.05$, $d / R=1.0), \operatorname{Fr}=0$.

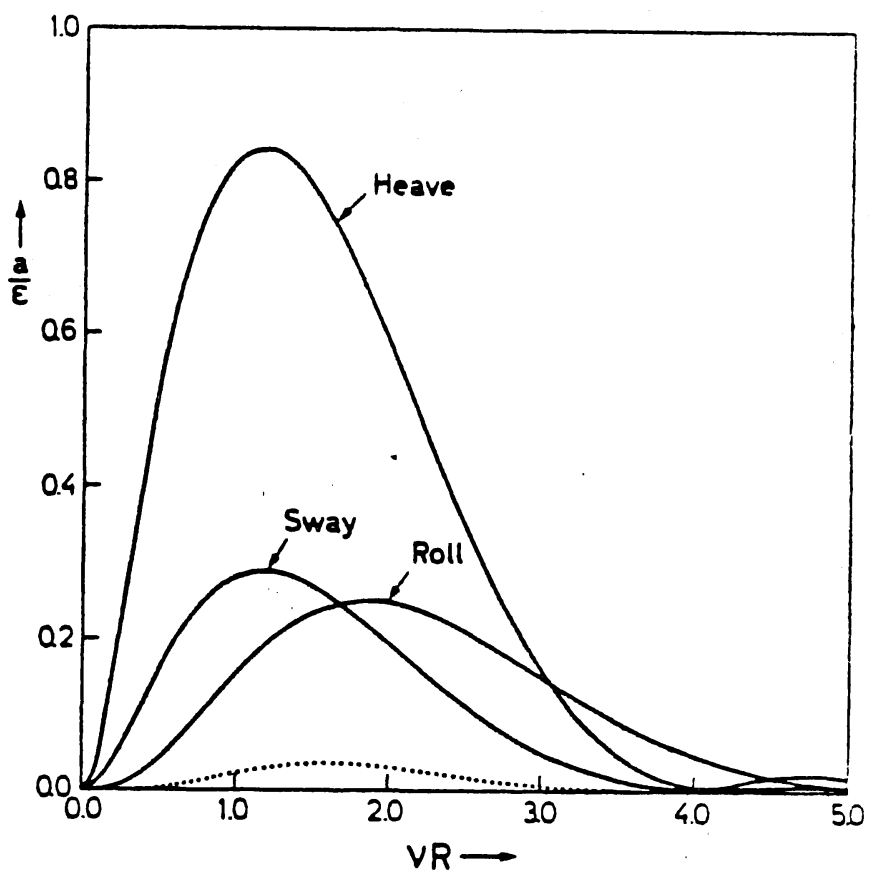

Fig. 3b. Amplitudes for radiated waves in heave, sway and roll ( $b / R=0.3$, $d / R=1.0)$, $F r=0$. The dotted curve is the damping force D/pgRe in roll.

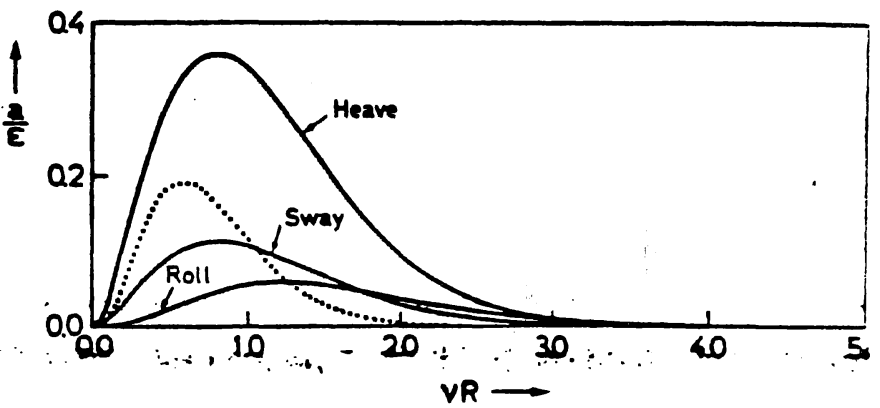

Fig. 4. Amplitudes for radiated waves in heave, sway and roll ( $b / R=0.3$, $\mathrm{d} / \mathrm{R}=2.0$ ), $\mathrm{Fr}=0$. The dotted curve is the damping force $\mathrm{D} / \rho \mathrm{gR} \varepsilon$ in heave. 


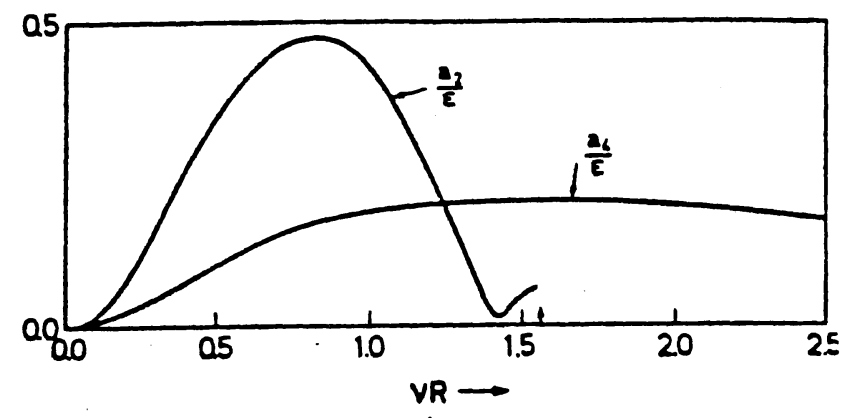

Fig. 5a.
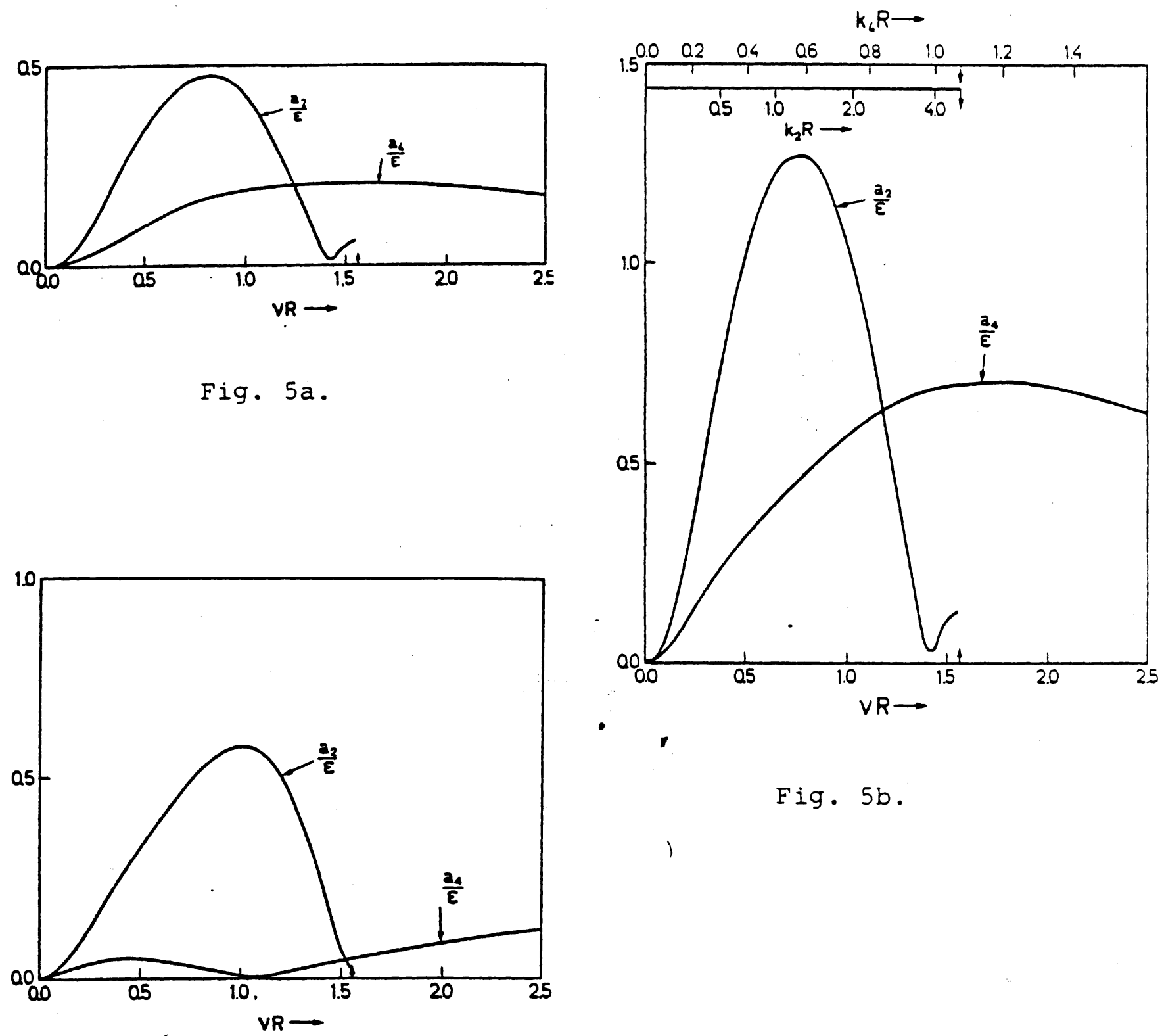

Fig. 5b.

Fig. 5c.

Figs 5 Amplitudes for radiated waves in sway (5a), heave (5b) and roll (5c) for $F r=0.2(b / R=0.3, d / R=1.0)$. The arrows at the $v$ R-axis indicate $\tau=1 / 4$. On $f$ ig. $5 \mathrm{~b}$ are indicated the values of the wave numbers $k_{2}$ and $k_{4}$. 


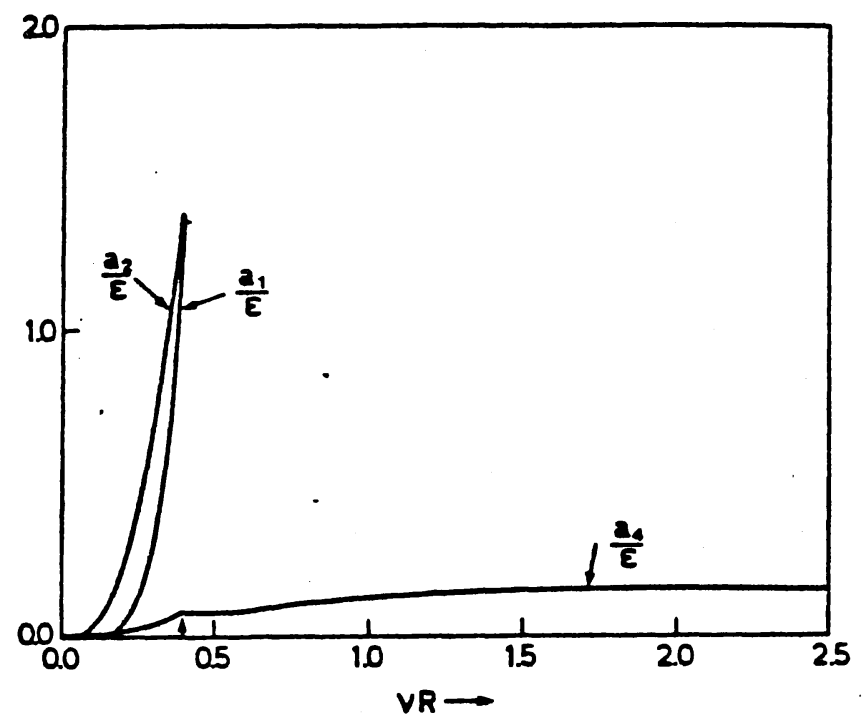

Fig. 6a.
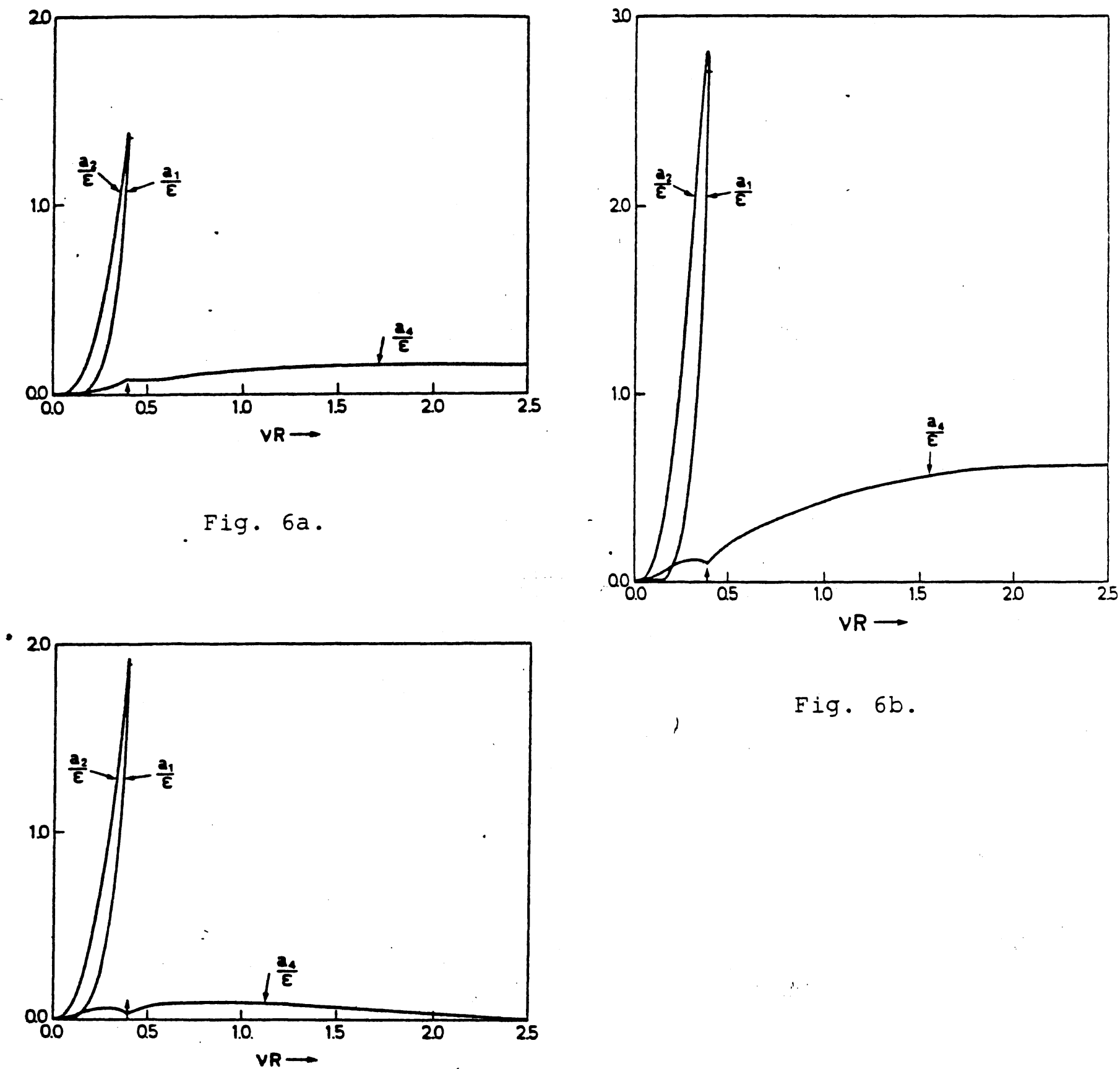

Fig. 6b.

Fig. 6c.

Figs 6 Amplitudes for radiated waves in sway (6a), heave (6b) and roll $(6 c)$ for $\mathrm{Fr}=0.4(\mathrm{~d} / \mathrm{R}=1.0)$. The arrows at the $v R-$ axis indicate $\tau=1 / 4$. 




Fig. 7. Damping force $D / \rho \jmath R \varepsilon$ and mean second order horizontal force $\overline{\mathrm{F}}_{\mathrm{x}} / \rho g \varepsilon^{2}$ in heave for $F r=0,0.2$ and 0.4 . The first arrow at the UR-axis indicates $\tau=1 / 4$ for $F r=0.4$ and the second arrow $\tau=1 / 4$ for $\operatorname{Fr}=0.2$.

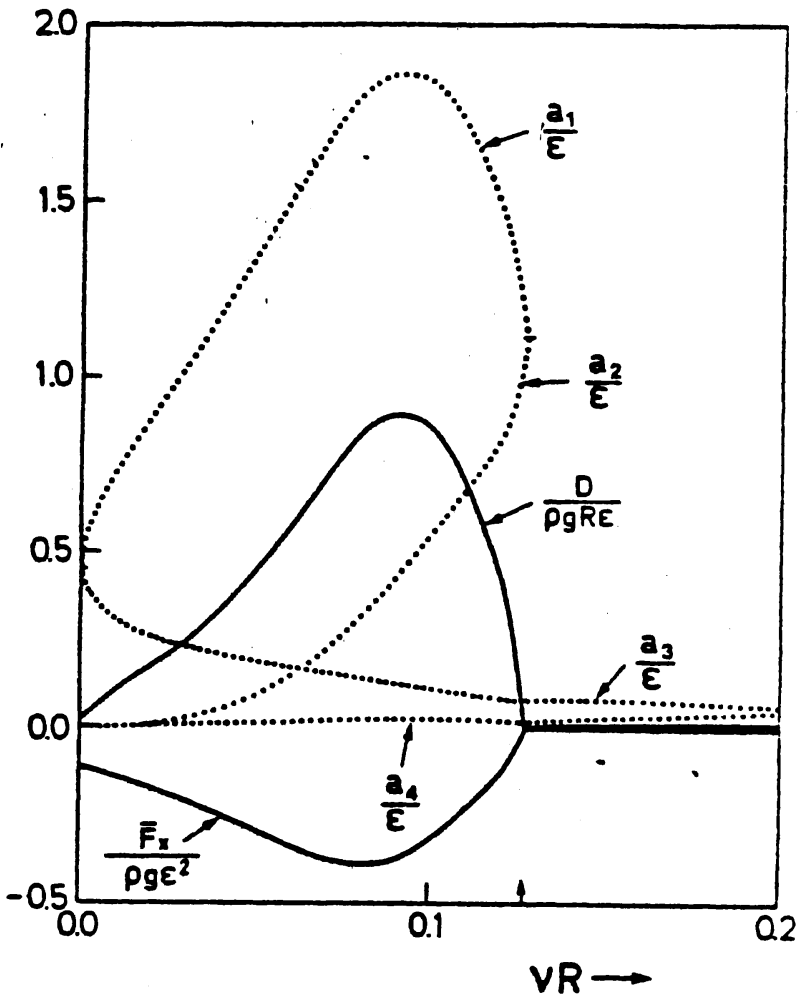

Fig. 8. Damping force $D / p g R \varepsilon$ and mean second order horizontal force $\overline{\mathrm{F}}_{\mathrm{X}} / \rho \mathrm{g \varepsilon}{ }^{2}$ in roll for Fr $=0.7$. Radiated amplitudes are dotted. The arrow at the UR-axis indicates $\tau=1 / 4$. 


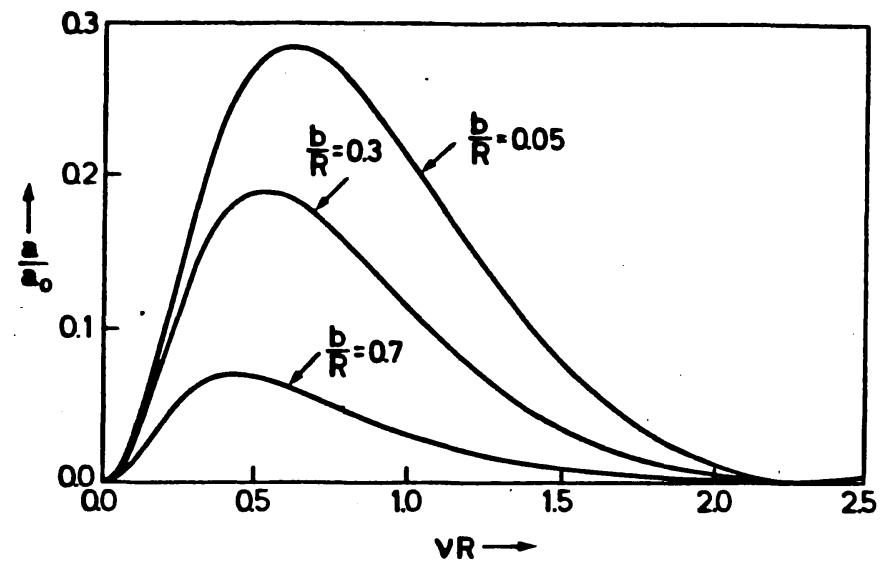

Fig. 9. Amplitudes of reflected waves in the diffraction problem for various values of $b / R(d / R=1.0), F r=0$.

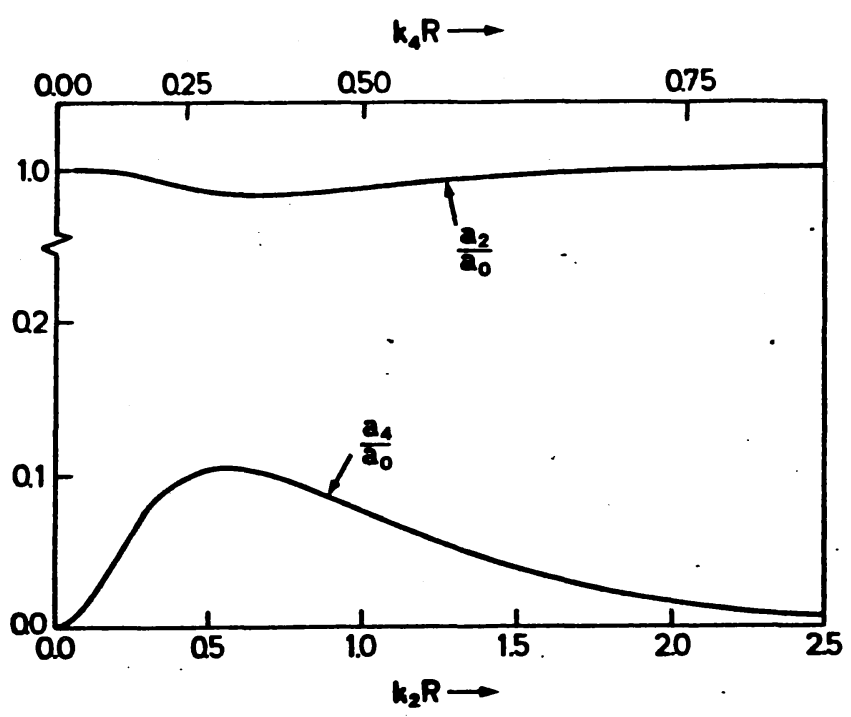

Fig. 10a. Amplitudes of the reflected $\mathrm{k}_{4}$ wave and transmitted $\mathrm{k}_{2}$ wave when a $\mathrm{k}_{2}$ wave ls incident upon the cylin$\operatorname{der}(b / R=0.3, d / R=1.0), F r=0.2$.



Fig. 10b. Amplitudes of the reflected $\mathrm{k}_{2}$ wave and transmitted $\mathrm{k}_{4}$ wave when a" $\mathrm{k}_{4}$ wave in incident upon the cyl in$\operatorname{der}(b / R=0.3, d / R=1.0), F r=0.2$. The arrows at the $k \mathrm{R}$-axis indicate $\tau=1 / 4$. 


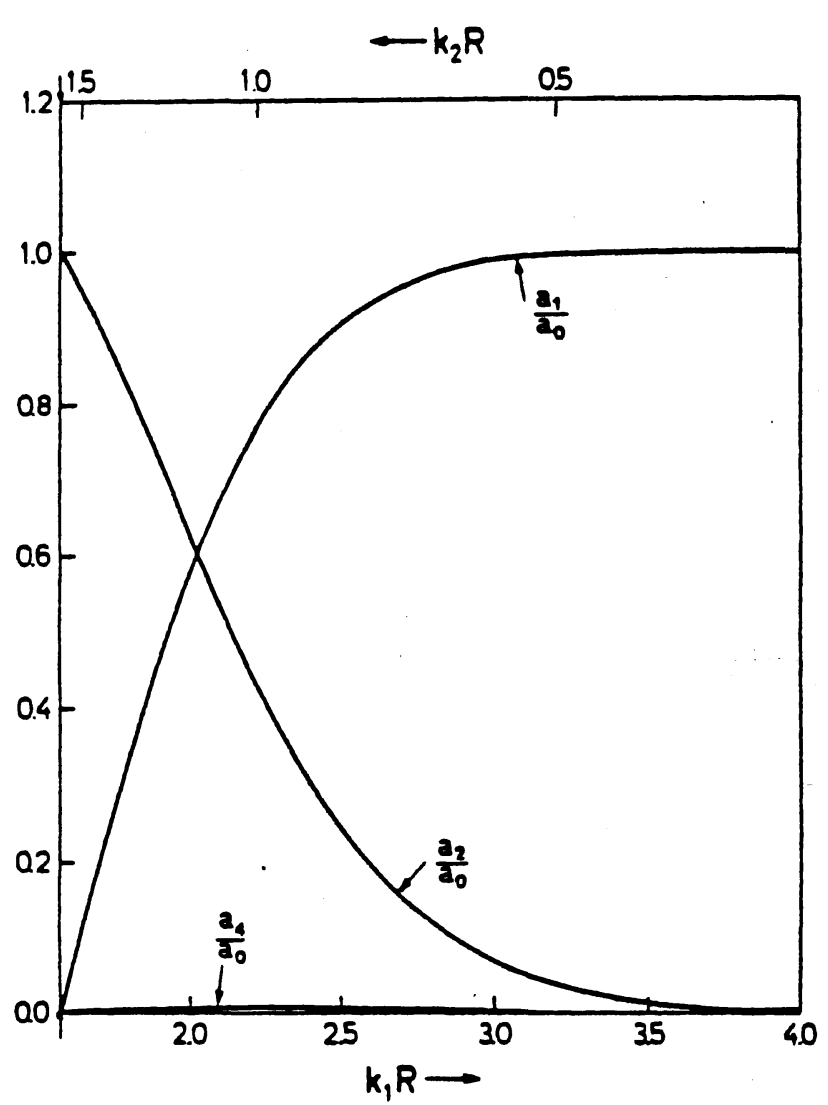

Fig. 11a. Amplitudes of the reflected $k_{2}$ wave, generated $k_{4}$ wave and transmitted $k_{1}$ wave when a $k_{1}$ wave is incident upon the cylinder $(b / R=0.3$, $d / R=1.0), F r=0.4$. The arrows at the $\mathrm{k} R$-axis indicate $\tau=1 / 4$.

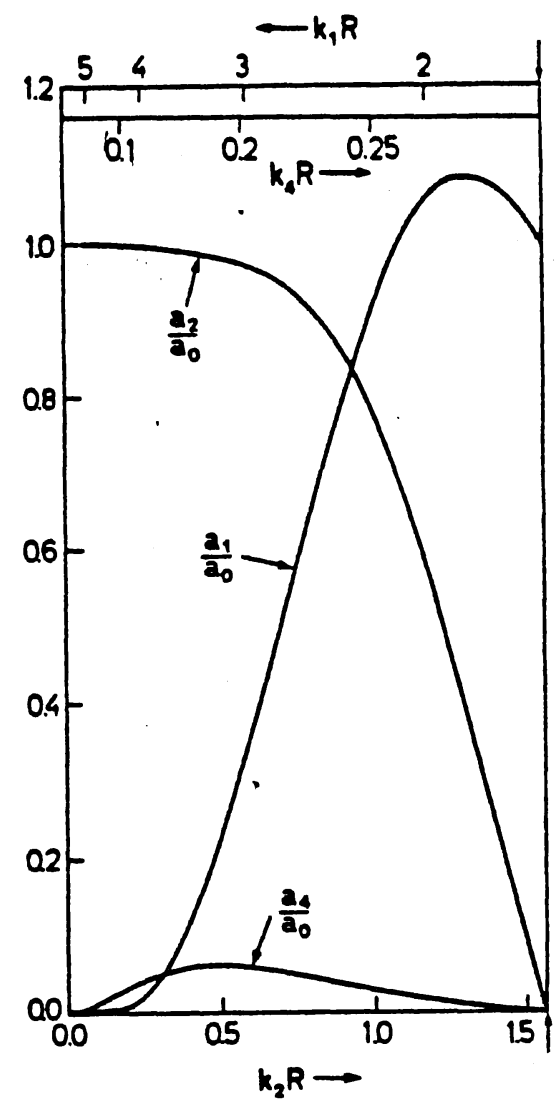

Fig. 11b. Amplitudes of the reflected $k_{4}$ wave and $k_{1}$ wave and transmitted $k_{2}$ wave when a $k_{2}$ wave is incident upon the cylin$\operatorname{der}(b / R=0.3, d / R=1.0), F r=0.4$. The arrows at the $k R$-axis indicate $\tau=1 / 4$. 


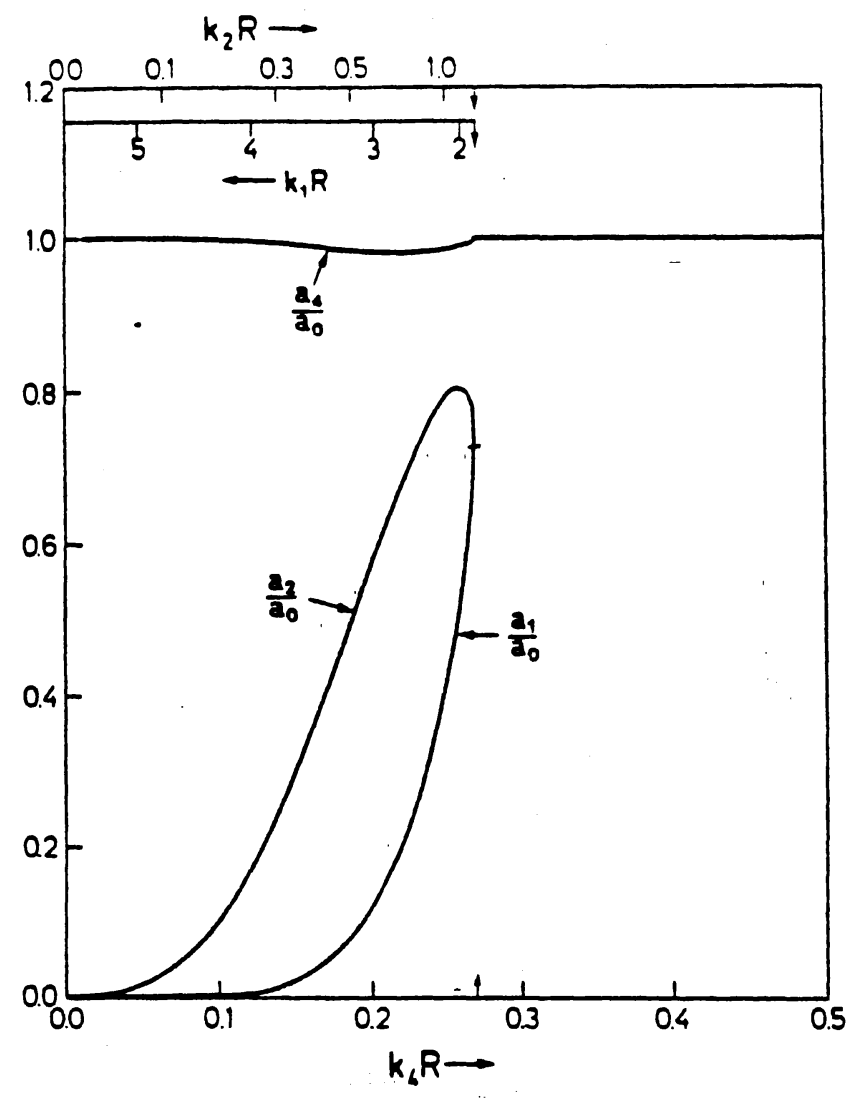

Fig. 11c. Amplitudes of the generated $k_{1}$ wave, reflected $k_{2}$ wave and transmitted $k_{4}$ wave when $a k_{4}$ wave is incident upon the cylinder $(b / R=0.3, d / R=1.0)$, Fr $=0.4$. The arrows at the $k R-a x i s$ indicate $\tau=1 / 4$.

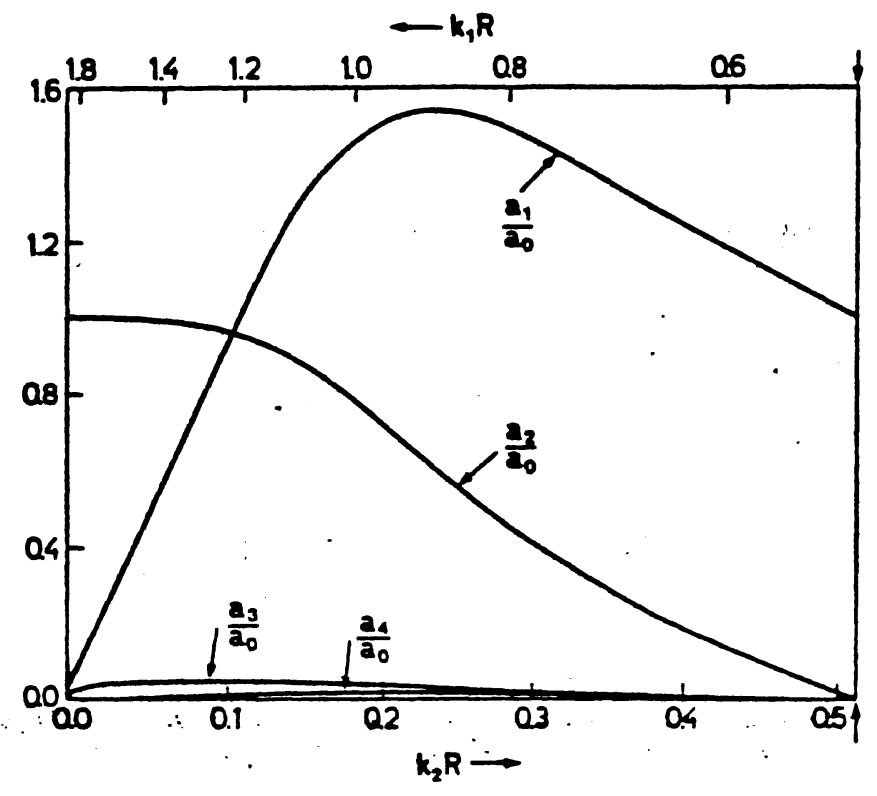

Fig. 12. Amplitudes of the reflected $k_{4}$ wave, $k_{1}$ wave and $k_{3}$ wave and transmitted $k_{2}$ wave when a $k_{2}$ wave is incident upon the $c y l$ inder $(b / R=0.3, d / B=1.05, F r=0.7$. The arrows at the $\mathrm{kP}$-axis indicate $\tau=1 / 4$. 




Fig. 13a. First order horizontal force $\mathrm{X}_{1} / \rho g \mathrm{Ra}_{0}$ and mean second order horizontal force $\overline{\mathrm{F}}_{\mathrm{x}} / \mathrm{\rho ga} \mathrm{O}_{0}^{2}$ for $\mathrm{Fr}=0,0.2$ and 0.4 with incoming wave travelling downstream $(b / R=0.3, d / R=1.0)$. For $F r=0.2$ and 0.4 the incoming wave is a $k_{4}$ wave.

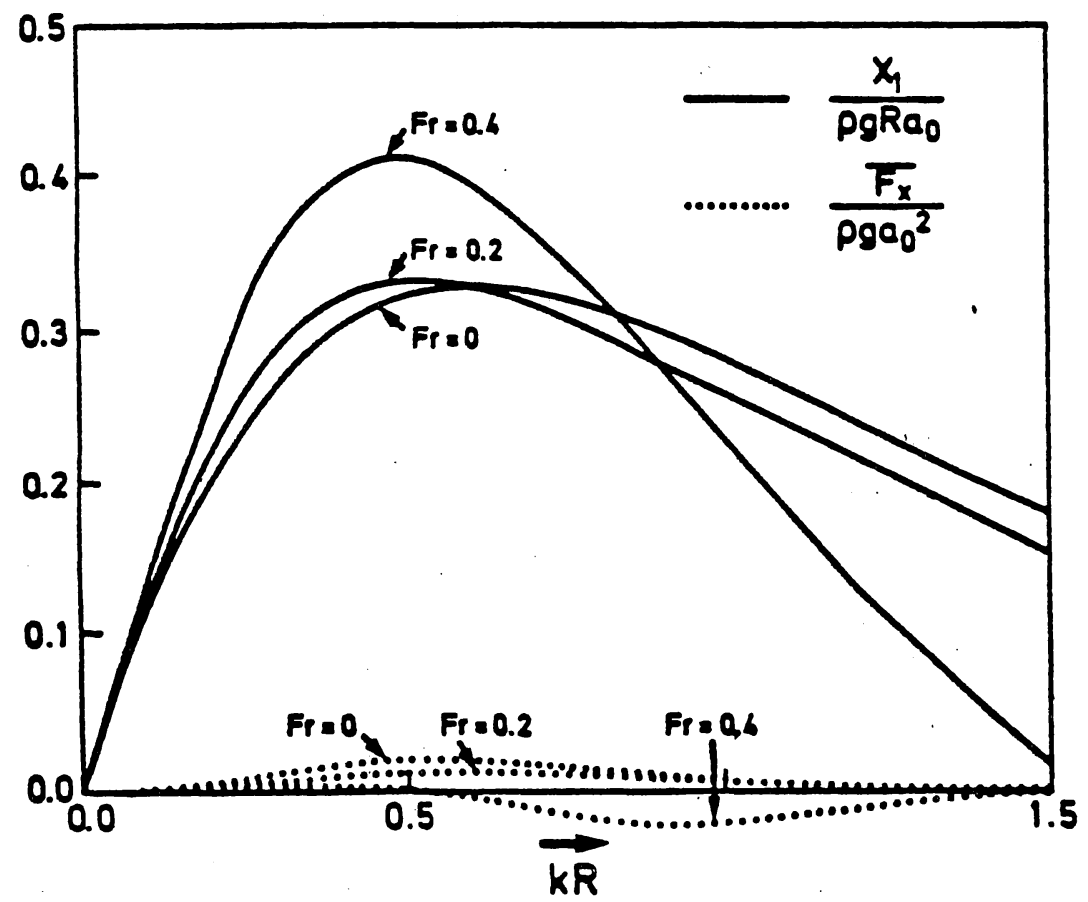

Fig. 13b. First order horizontal force $\mathrm{X}_{1} / \mathrm{kgRa} \mathrm{a}_{0}$ and mean second order horizontal force $\overline{\mathrm{F}}_{\mathrm{X}} / \rho \mathrm{ga}_{0}^{2}$ for $\mathrm{Fr}=0,0.2$ and 0.4 with incoming wave travelling upstream $(b / R=0.3, d / R=1.0)$. For $F r=0.2$ and 0.4 the incoming wave is a $k_{2}$ wave. 


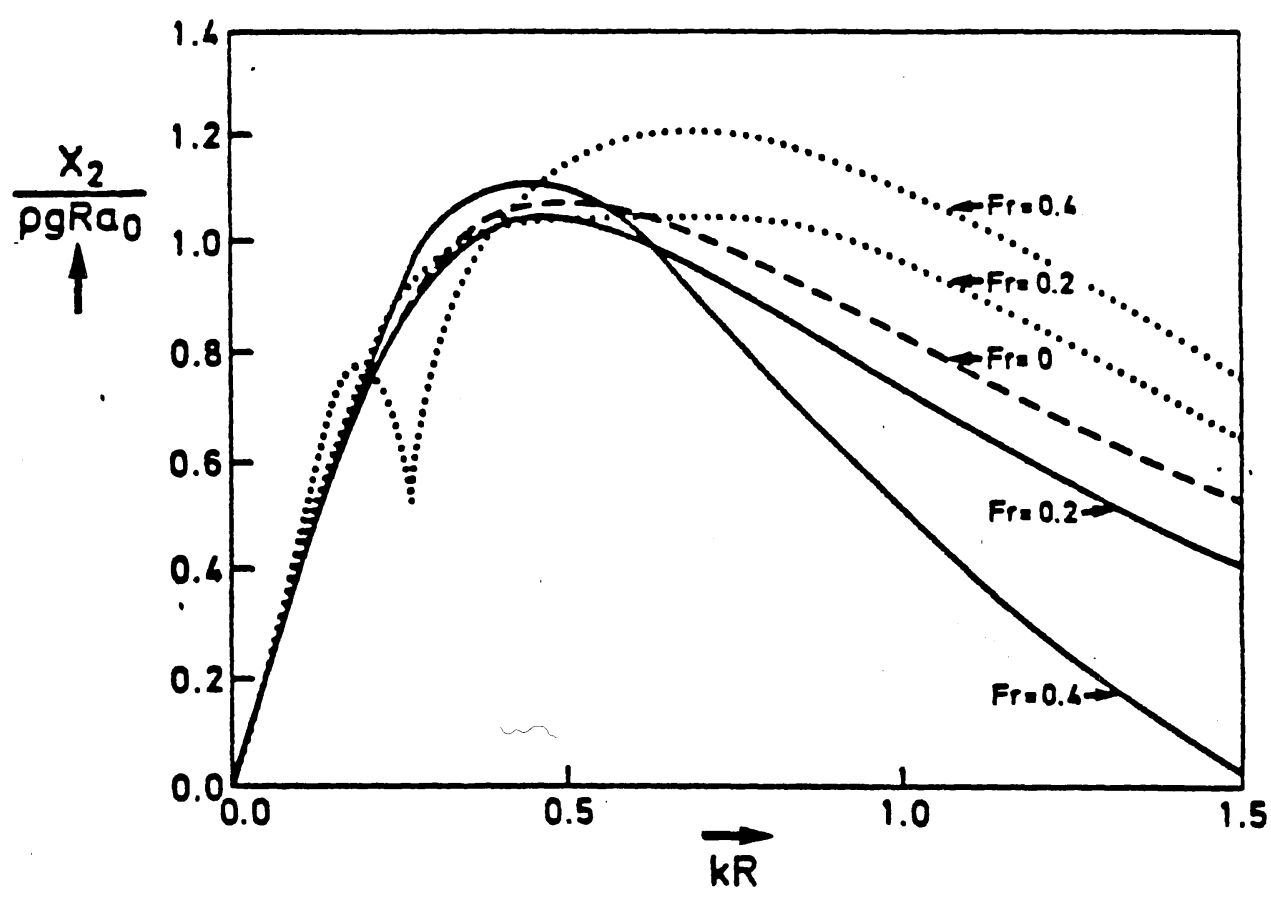

Fig. 13c. First order vertical force $\mathrm{X}_{2} / \rho g \mathrm{Ra}_{0}$ for $\mathrm{Fr}=0$ (---), for $\mathrm{Fr}=0.2$ and 0.4 with incoming wave travelling downstream $(\cdots)$ and for $F r=0.2$ and 0.4 with incoming wave travelling upstream (-), $(b / R=0.3, d / R=1.0)$.

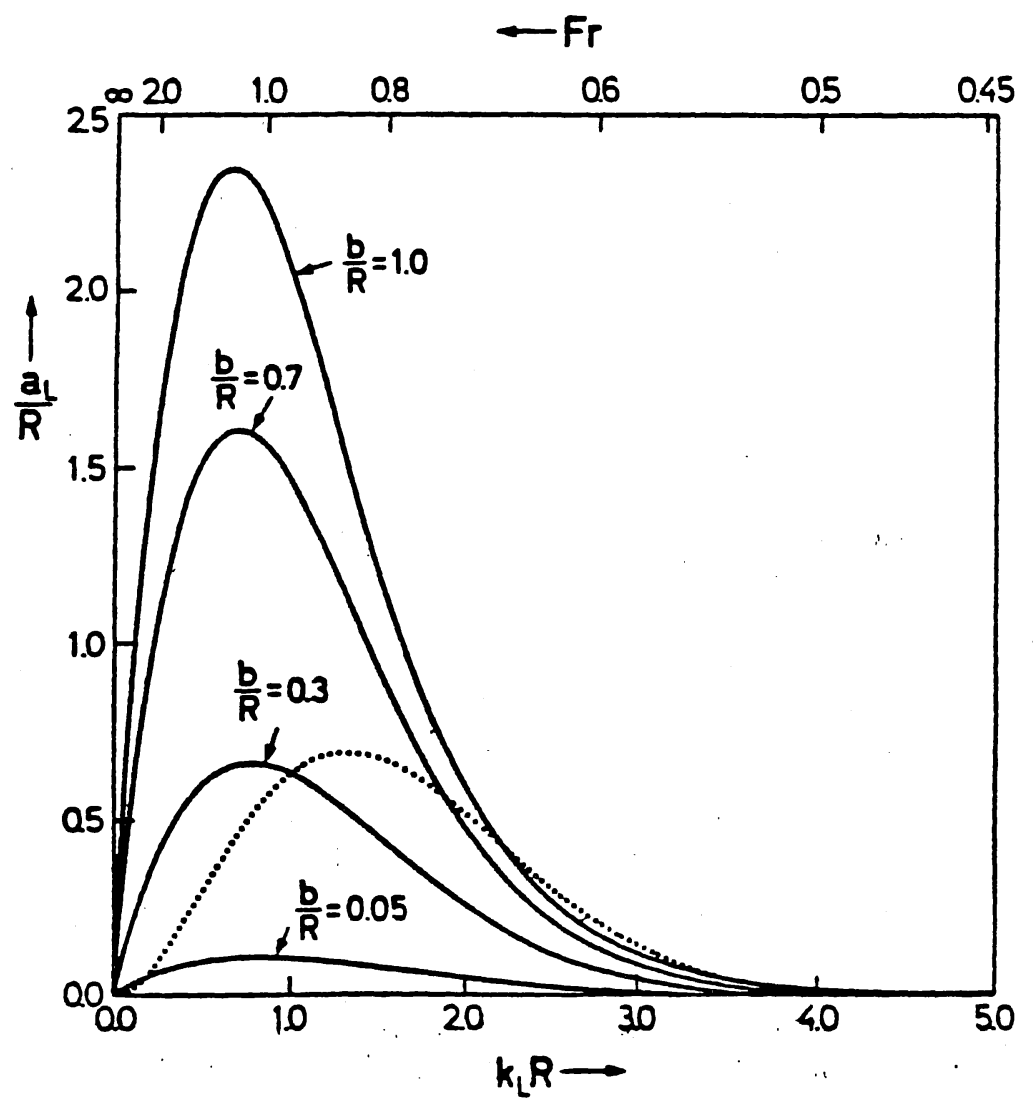

Fig. 14. Amplitude of the lee-wave at $X=-\infty$ for various values of $b / R$ $(d / R=1.0)$. The lee-wave number $k_{t}$ is given by $k_{L}=g / U^{2}=1 / F r^{2} R$. The dotted curve is $\max |\partial x / \partial x|$ at $x=-\infty$ for $b / R=0.3(d / R=1.0)$. 